\title{
Applying the Influence of Conformational Freedom on the Network Topologies Showing Impressive Proton Conductivity
}

Avik De, ${ }^{\text {a Olivia Basu, }}{ }^{\text {b Tannistha Basu, }}{ }^{\text {a }}$ Raju Mondal*a

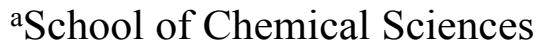

Indian Association for the Cultivation of Science,

2A \& 2B, Raja S. C. Mullick Road, Kolkata 700032,

West Bengal, India

bSchool of Chemistry

University of Hyderabad,

Hyderabad 500046,

Telengana, India

*Email: icrm@iacs.res.in 


\section{Table of contents}

Figure. S1. The structure diagram of ligands

Figure. S2. FT-IR spectra of PDA-FA

Figure. S3. FT-IR spectra of PDA-WC

Table S1. Crystallographic table

Figure. S4. Picture of pelletized sample with carbon coating attached on both sides

Table S2. Some selected bond lengths $(\AA)$ and bond angles $\left(^{\circ}\right)$ for PDA-WC

Table S3. Some selected bond lengths $(\AA)$ and bond angles $\left(^{\circ}\right)$ for PDA-FA

Table S4. Some selected bond lengths $(\AA)$ and bond angles $\left(^{\circ}\right)$ for $[\mathbf{Z n}(\mathbf{P D A})]_{n}$

Figure. S5. Crystal structure of $[\mathbf{Z n}(\mathbf{P D A})]_{\mathbf{n}}$ showing 2D pillared layered network

Figure. S6. $\mathrm{N}_{2}$ gas adsorption by PDA-FA at $77 \mathrm{~K}$

Figure. S7. $\mathrm{N}_{2}$ gas adsorption by PDA-WC at $77 \mathrm{~K}$

Figure. S8. TGA plot of PDA-FA

Figure. S9. Variable temperature PXRD data plot for PDA-FA

Figure. S10. Comparison of FT-IR spectra at various conditions for PDA-FA

Figure. S11. Variable temperature PXRD data plot for PDA-WC

Figure. S12. TGA plot of PDA-WC

Figure. S13. Comparison of FT-IR spectra at various conditions for PDA-WC

Table S5. Comparison of the Proton Conductivities of PDA-FA \& PDA-WC with other Nonporous Proton-Conducting Materials.

Figure. S14. Nyquist plots of impedance spectra of PDA-FA at $40^{\circ} \mathrm{C}$ and $70^{\circ} \mathrm{C}$ (at $33 \% \mathrm{RH}$ )

Figure. S15. Nyquist plots of impedance spectra of PDA-FA at $40^{\circ} \mathrm{C}$ and $70^{\circ} \mathrm{C}$ (at $75 \% \mathrm{RH}$ )

Figure. S16. Nyquist plots of impedance spectra of PDA-WC at $40^{\circ} \mathrm{C}$ and $70^{\circ} \mathrm{C}$ (at $33 \% \mathrm{RH}$ ) 
Figure. S17. Nyquist plots of impedance spectra of PDA-WC at $40^{\circ} \mathrm{C}$ and $70^{\circ} \mathrm{C}$ (at $75 \% \mathrm{RH}$ )

Figure. S18. Nyquist plots of impedance spectra of activated PDA-WC at $40^{\circ} \mathrm{C}$ and $70^{\circ} \mathrm{C}$ (at $33 \% \mathrm{RH})$

Figure. S19. Nyquist plots of impedance spectra of activated PDA-WC at $40^{\circ} \mathrm{C}$ and $70^{\circ} \mathrm{C}$ (at $75 \% \mathrm{RH})$

Figure. S20. Nyquist plots of impedance spectra of activated PDA-WC at $40^{\circ} \mathrm{C}, 50^{\circ} \mathrm{C}, 60^{\circ} \mathrm{C}$, $70^{\circ} \mathrm{C}$ and $80^{\circ} \mathrm{C}$ (at $\left.90 \% \mathrm{RH}\right)$

\section{References}



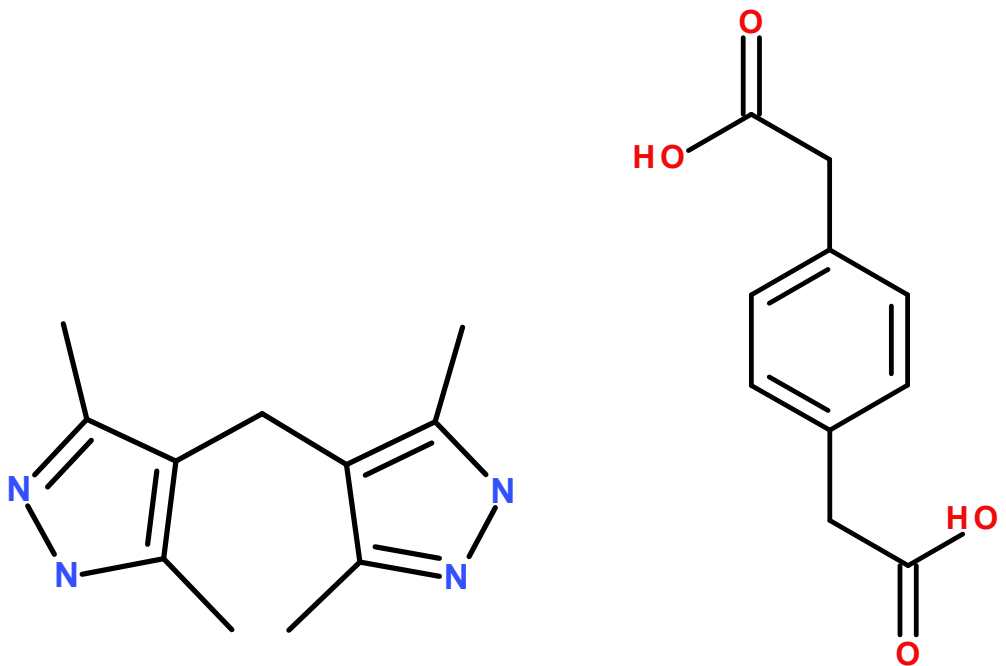

$\mathrm{H}_{2}$ MDP

1,4-phenylene diacetic acid ( H $_{2}$ PDA)

Figure. S1. The structure diagram of ligands

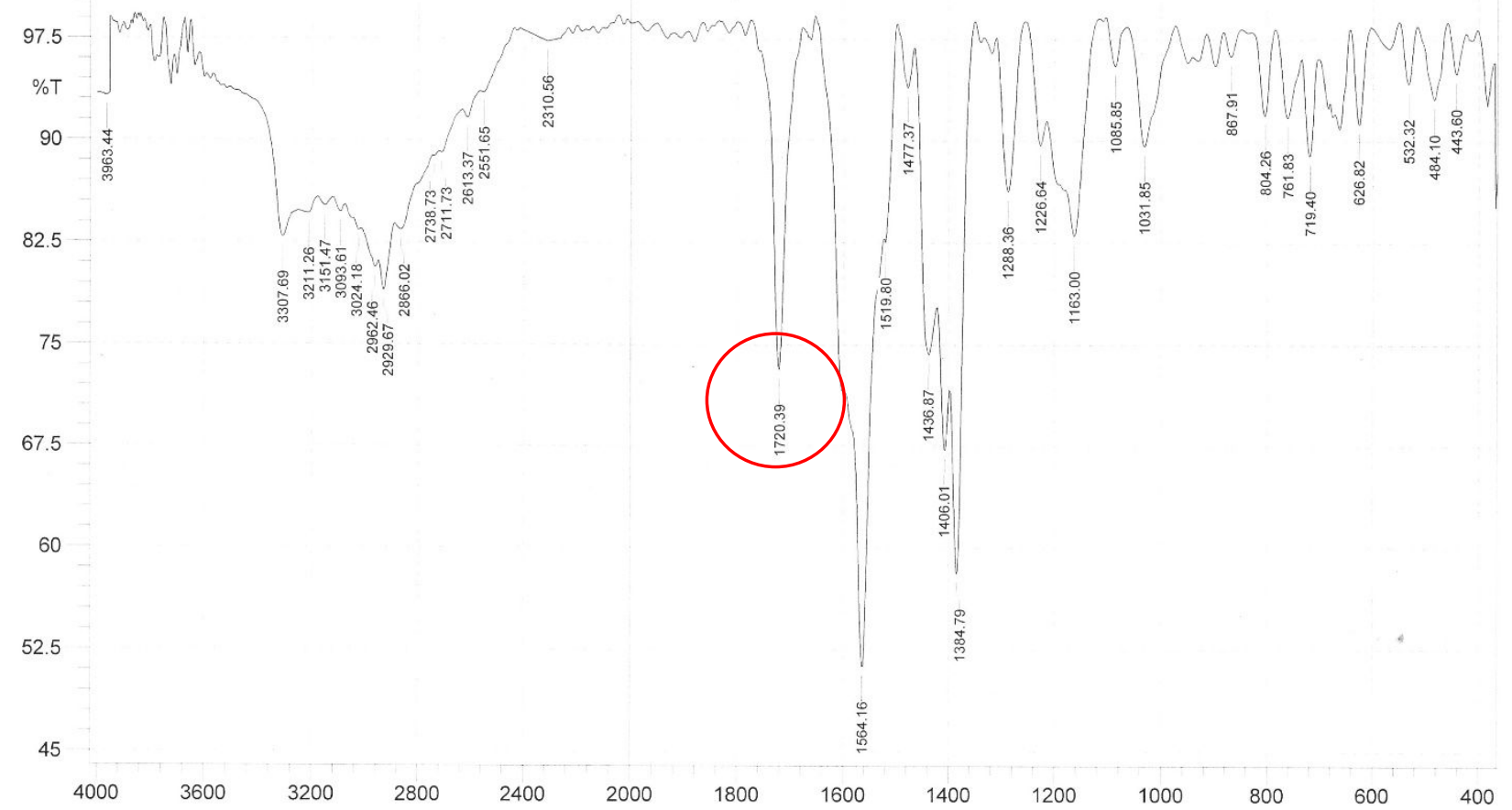

Figure. S2. FT-IR spectra of PDA-FA (red circled peak at $1720 \mathrm{~cm}^{-1}$ is for C-O of free carboxylic acid groups.) 


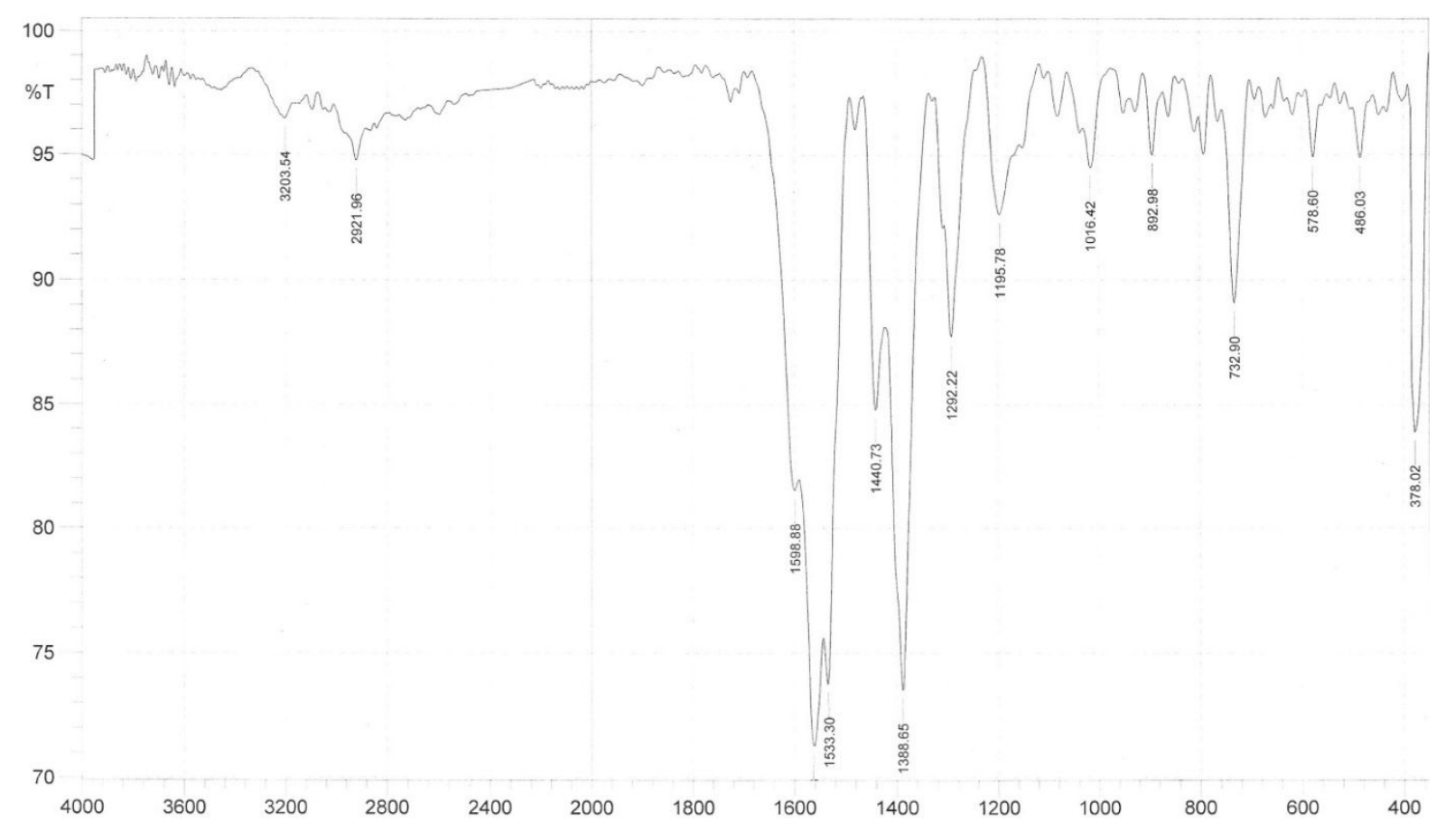

Figure. S3. FT-IR spectra of PDA-WC 
Table S1. Crystallographic table

\begin{tabular}{|c|c|c|c|}
\hline & PDA-WC & PDA-FA & {$[\mathrm{Zn}(\mathrm{PDA})]_{\mathrm{n}}$} \\
\hline $\begin{array}{l}\text { Empirical } \\
\text { formula }\end{array}$ & $\mathrm{C}_{22} \mathrm{H}_{38} \mathrm{~N}_{4} \mathrm{O}_{10} \mathrm{Zn}$ & $\mathrm{C}_{26} \mathrm{H}_{29} \mathrm{~N}_{4} \mathrm{O}_{6} \mathrm{Zn}$ & $\mathrm{C}_{10} \mathrm{H}_{8} \mathrm{O}_{4} \mathrm{Zn}$ \\
\hline $\begin{array}{c}\text { Formula } \\
\text { weight }\end{array}$ & 583.93 & 558.90 & 257.53 \\
\hline $\begin{array}{l}\text { Crystal } \\
\text { system }\end{array}$ & Monoclinic & Triclinic & Monoclinic \\
\hline $\begin{array}{l}\text { Space } \\
\text { group }\end{array}$ & $\mathrm{C} 2 / \mathrm{c}$ & $\mathrm{P} \overline{1}$ & $\mathrm{P} 2{ }_{1} / \mathrm{c}$ \\
\hline$a / \AA$ & $27.6934(8)$ & $9.7141(5)$ & $19.6593(12)$ \\
\hline$b / \AA$ & $11.4193(3)$ & $12.2458(6)$ & $4.8783(3)$ \\
\hline$c / \AA \AA$ & $18.3753(5)$ & $12.5115(6)$ & $9.6388(6)$ \\
\hline$\alpha /^{\circ}$ & 90.00 & $88.179(1)$ & 90.00 \\
\hline$\beta /{ }^{\circ}$ & $115.3680(10)$ & $67.770(1)$ & $95.209(2)$ \\
\hline$\gamma /{ }^{\circ}$ & 90.00 & $70.806(1)$ & 90.00 \\
\hline$V / \AA^{3}$ & $5250.7(3)$ & $1293.80(11)$ & $920.58(10)$ \\
\hline $\begin{array}{l}\text { Reflection } \\
\text { collected }\end{array}$ & 35176 & 12890 & 11722 \\
\hline $\begin{array}{l}\text { Unique } \\
\text { reflections }\end{array}$ & 6293 & 5288 & 2212 \\
\hline $\begin{array}{l}\text { Observed } \\
\text { reflections }\end{array}$ & 4680 & 4420 & 1667 \\
\hline \multicolumn{4}{|l|}{$[I>2 \sigma(I)]$} \\
\hline$R_{1}$ & 0.0763 & 0.0353 & 0.0370 \\
\hline$w R_{2}$ & 0.2177 & 0.0761 & 0.0862 \\
\hline CCDC numbers & 1024079 & 689714 & 1024078 \\
\hline
\end{tabular}




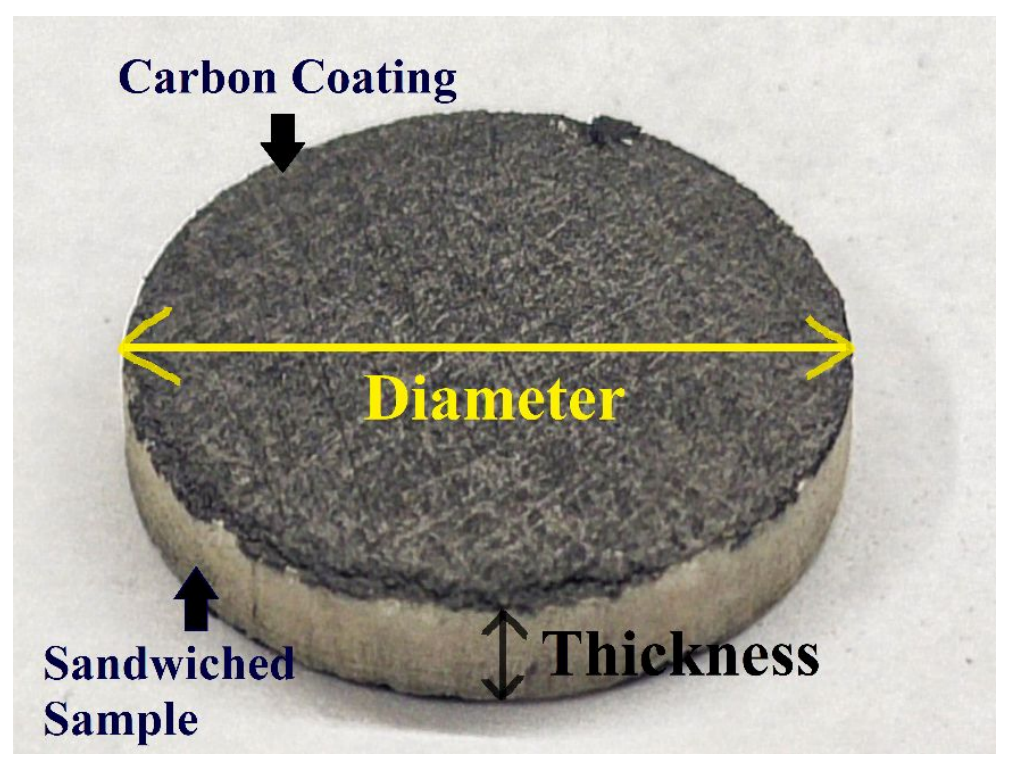

Figure. S4. Picture of pelletized sample with carbon coating attached on both sides

Table S2. Some selected bond lengths $(\AA)$ and bond angles $\left(^{\circ}\right)$ for PDA-WC

\begin{tabular}{|c|c|c|c|c|c|}
\hline \multicolumn{2}{|c|}{ Bond lengths(Å) } & \multicolumn{4}{|c|}{ Bond angles $\left({ }^{\circ}\right)$} \\
\hline Zn1-O3 & $1.955(3)$ & O3-Zn1-O1 & $113.18(13)$ & C1-N1-Zn1 & $129.9(3)$ \\
\hline Zn1-O1 & $1.979(3)$ & O3-Zn1-N3 & $115.62(13)$ & N2-N1-Zn1 & $125.5(3)$ \\
\hline Zn1-N3 & $2.008(3)$ & O1-Zn1-N3 & $113.01(14)$ & C12-O1-Zn1 & $109.9(3)$ \\
\hline Zn1-N1 & $2.026(3)$ & O3-Zn1-N1 & $108.33(14)$ & C4-N3-Zn1 & $133.5(3)$ \\
\hline & & O1-Zn1-N1 & $99.19(13)$ & N4-N3-Zn1 & $120.3(3)$ \\
\hline & & N3-Zn1-N1 & $105.76(14)$ & $\mathrm{C} 21-\mathrm{O} 3-\mathrm{Zn} 1$ & $127.9(3)$ \\
\hline
\end{tabular}


Table S3. Some selected bond lengths $(\AA)$ and bond angles $\left(^{\circ}\right)$ for PDA-FA.

\begin{tabular}{|c|c|c|c|c|c|c|c|}
\hline \multicolumn{2}{|c|}{ Bond lengths $(\AA)$} & \multicolumn{6}{|c|}{ Bond angles $\left({ }^{\circ}\right)$} \\
\hline Zn1-O5 & $1.9423(15)$ & O5-Zn1-O1 & $111.91(7)$ & O1-Zn1-N1 & $102.76(7)$ & C1-N1-Zn1 & $131.90(14)$ \\
\hline Zn1-O1 & $1.9600(15)$ & O5-Zn1-N3 & $110.88(7)$ & N3-Zn1-N1 & $105.40(7)$ & N2-N1-Zn1 & $119.71(14)$ \\
\hline Zn1-N3 & $2.0145(18)$ & O1-Zn1-N3 & $111.87(7)$ & C12-O1-Zn1 & $117.08(15)$ & C7-N3-Zn1 & $130.21(15)$ \\
\hline Zn1-N1 & $2.0153(18)$ & O5-Zn1-N1 & $113.62(7)$ & C22-O5-Zn1 & $126.00(15)$ & N4-N3-Zn1 & $124.40(13)$ \\
\hline
\end{tabular}

Table S4. Some selected bond lengths $(\AA)$ and bond angles $\left(^{\circ}\right)$ for $[\mathbf{Z n}(\mathbf{P D A})]_{n}$

\begin{tabular}{|l|l|l|l|l|l|}
\hline \multicolumn{2}{|c|}{ Bond lengths( } & \multicolumn{3}{c|}{ Bond angles ( $)$} \\
\hline Zn1-O1 & $1.943(2)$ & O1-Zn1-O2 & $112.88(9)$ & O3-Zn1-O4 & $102.28(10)$ \\
\hline Zn1-O2 & $1.949(2)$ & O1-Zn1-O3 & $111.08(9)$ & C7-O4-Zn1 & $130.5(2)$ \\
\hline Zn1-O3 & $1.950(2)$ & O2-Zn1-O3 & $111.58(10)$ & C4-O3-Zn1 & $133.6(2)$ \\
\hline Zn1-O4 & $1.965(2)$ & O1-Zn1-O4 & $110.09(9)$ & C7-O2-Zn1 & $119.54(19)$ \\
\hline & & O2-Zn1-O4 & $108.38(9)$ & C4-O1-Zn1 & $120.33(19)$ \\
\hline
\end{tabular}




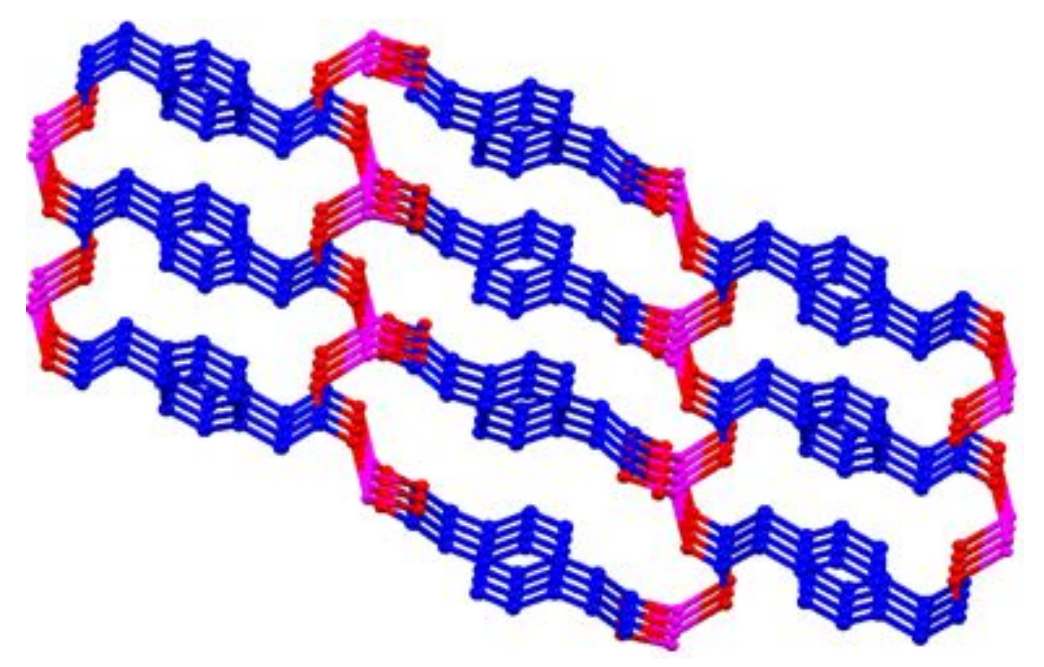

Figure. S5. Crystal structure of $[\mathbf{Z n}(\mathbf{P D A})]_{\mathbf{n}}$ showing 2D pillared layered network

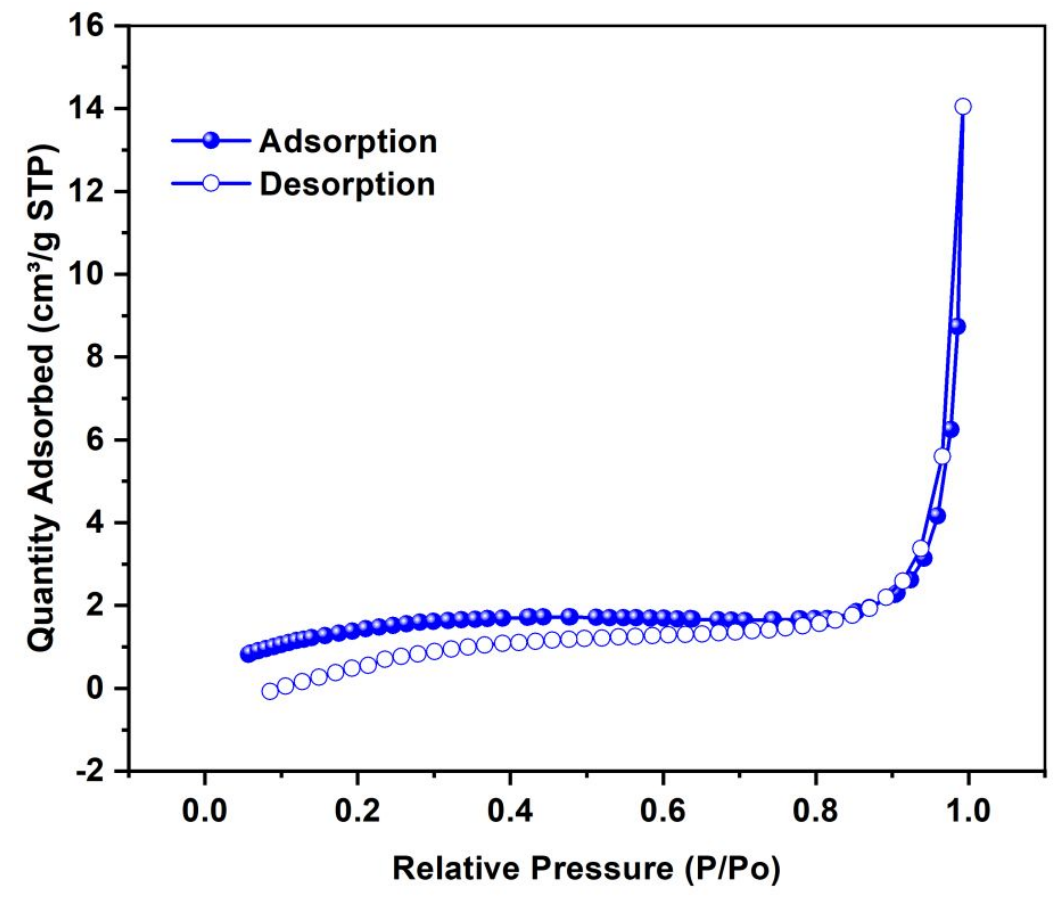

Figure. S6. $\mathrm{N}_{2}$ gas adsorption by PDA-FA at $77 \mathrm{~K}$ 


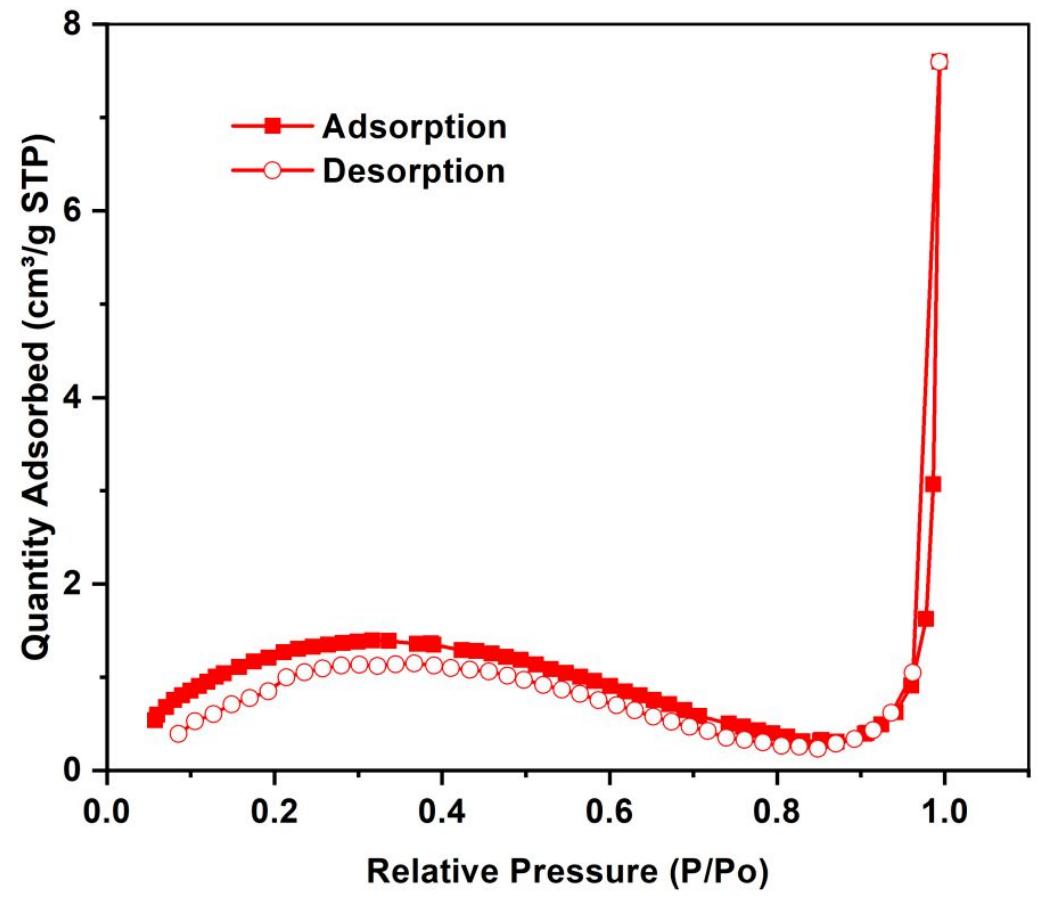

Figure. S7. $\mathrm{N}_{2}$ gas adsorption by PDA-WC at $77 \mathrm{~K}$



Figure. S8. TGA plot of PDA-FA (23\% mass loss occurs due to the decarboxylation of free carboxylic acid groups) 


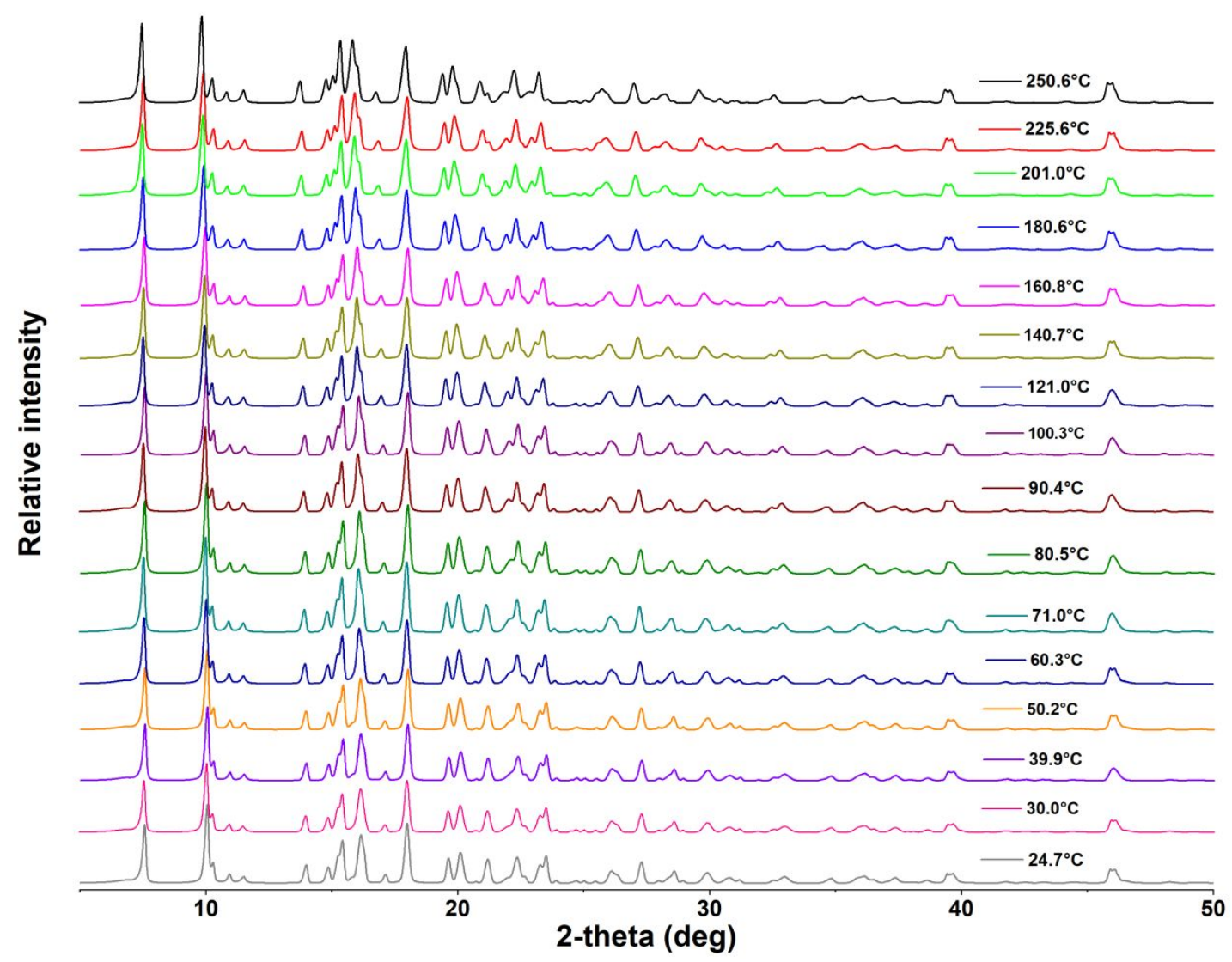

Figure. S9. Variable temperature PXRD data plot for PDA-FA

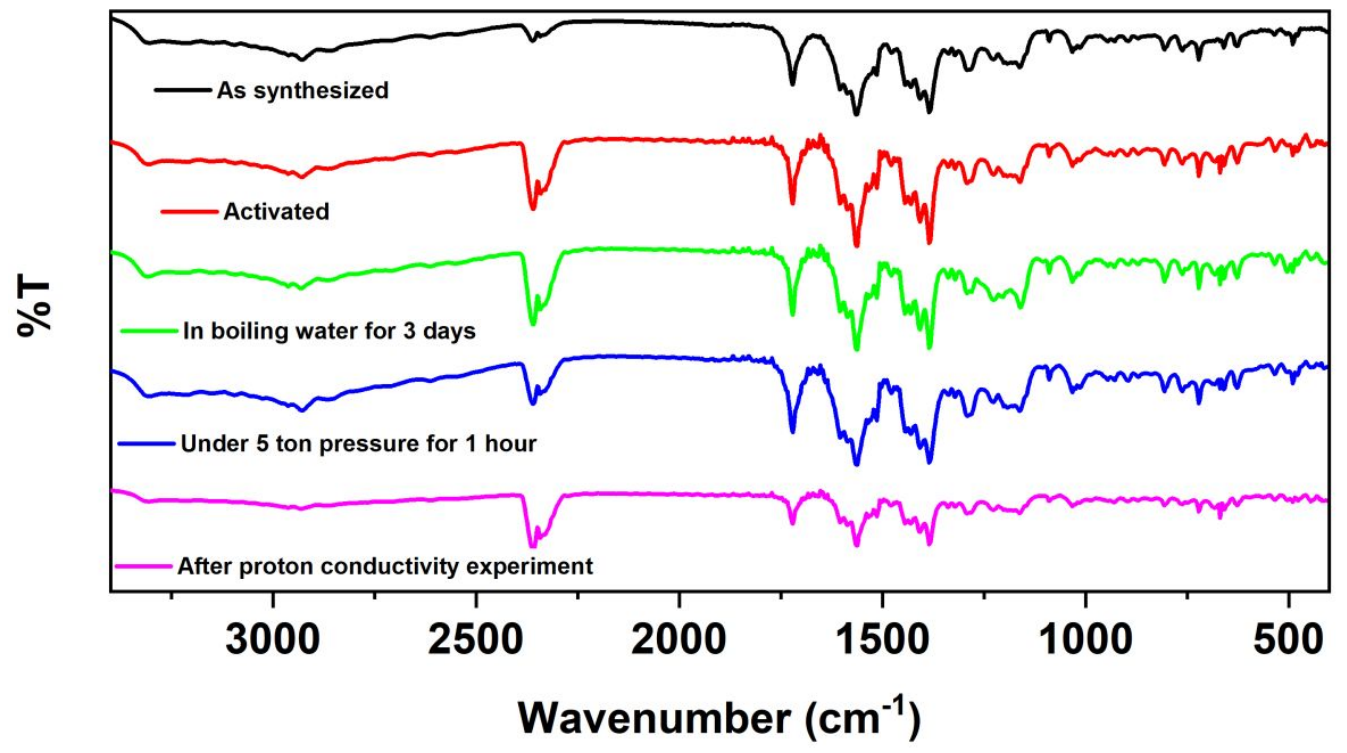

Figure. S10. Comparison of FT-IR spectra at various conditions for PDA-FA 


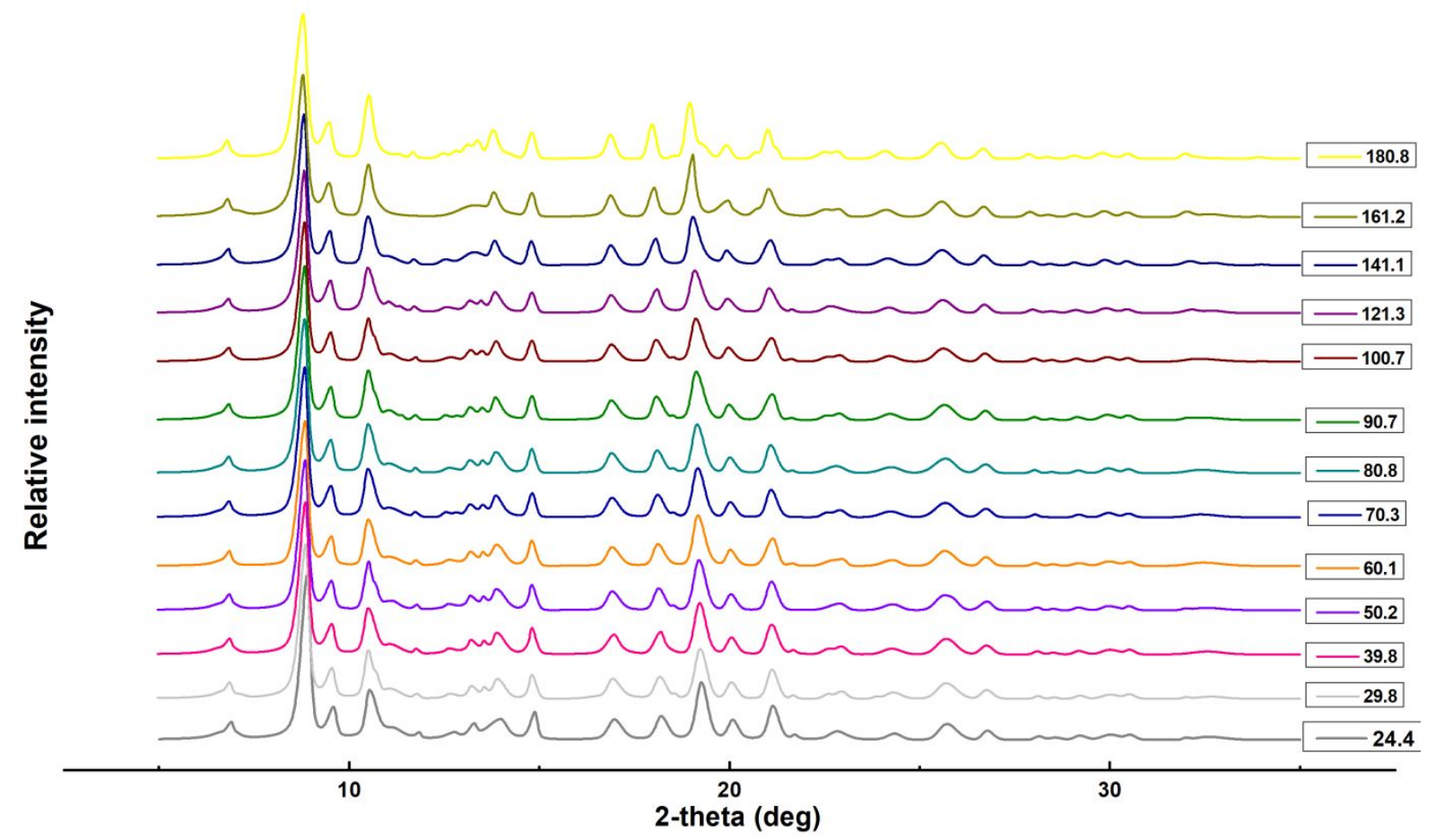

Figure. S11. Variable temperature PXRD data plot for PDA-WC

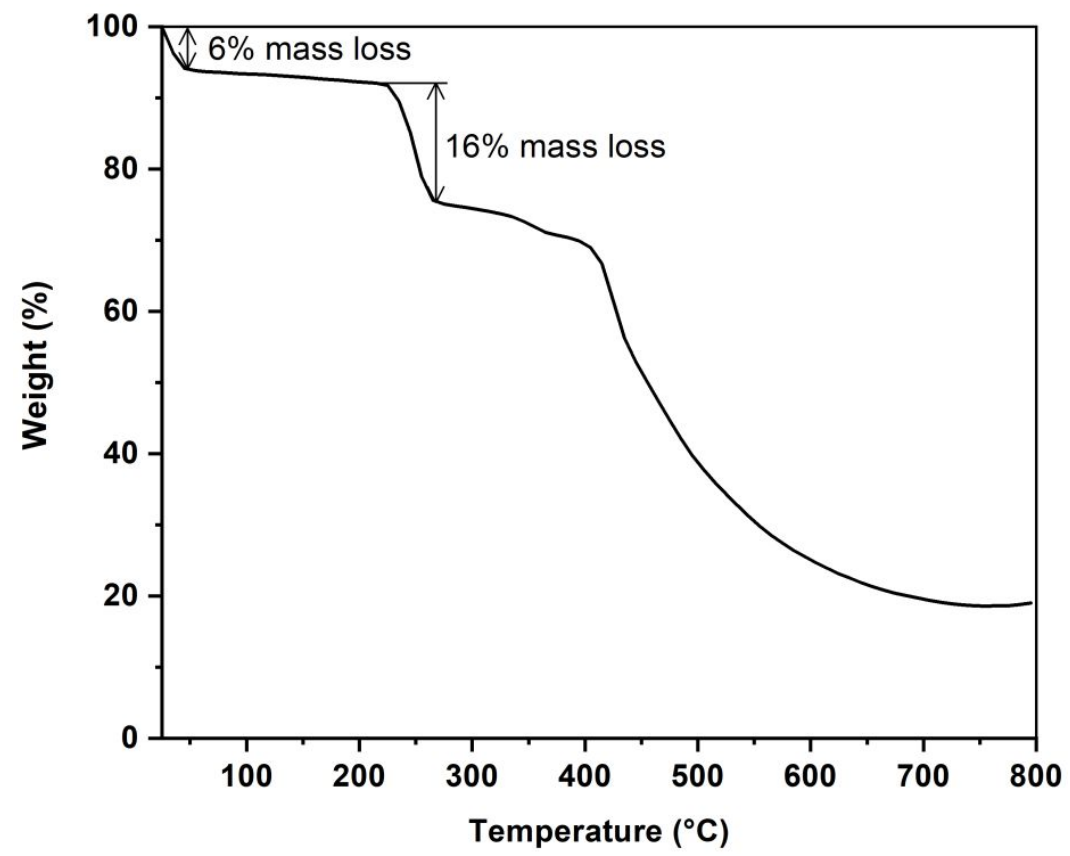

Figure. S12. TGA plot of PDA-WC (6\% mass loss occurs due to release of methanol molecules from MOF pores, $16 \%$ mass loss occurs due to the release of water molecules from MOF pores) 




Figure. S13. Comparison of FT-IR spectra at various conditions for PDA-WC 
Table S5. Comparison of the Proton Conductivities of PDA-FA \& PDA-WC with other Nonporous Proton-Conducting Materials.

\begin{tabular}{|c|c|c|c|}
\hline materials & conductivity (Scm-1) & testing conditions & Ref. \\
\hline$\left(\mathrm{C}_{2} \mathrm{~N}_{2} \mathrm{H}_{10}\right)_{0.5} \mathrm{CoPO}_{4}$ & $2.05 \times 10^{-3}$ & $56{ }^{\circ} \mathrm{C}$ and $98 \% \mathrm{RH}$ & 1 \\
\hline$[\mathrm{Zn}(p-\mathrm{IPhHIDC})]_{\mathrm{n}}$ & $1.9 \times 10^{-3}$ & $100{ }^{\circ} \mathrm{C}$ and $98 \% \mathrm{RH}$ & 2 \\
\hline$\left[\mathrm{Ni}_{3}(\mathrm{HL})_{2}\left(\mathrm{H}_{2} \mathrm{O}\right)_{10}\right] \cdot 4 \mathrm{H}_{2} \mathrm{O}$ & $1.43 \times 10^{-3}$ & $4{ }^{\circ} \mathrm{C}$ and $100 \% \mathrm{RH}$ & 3 \\
\hline$[\mathrm{Co}(p-\mathrm{IPhHIDC})]_{\mathrm{n}}$ & $1.07 \times 10^{-3}$ & $100^{\circ} \mathrm{C}$ and $98 \% \mathrm{RH}$ & 2 \\
\hline$\left[\mathrm{Zn}(\mathrm{PDA})_{0.5}(\mathrm{HPDA})\left(\mathrm{H}_{2} \mathrm{MDP}\right)\right]_{\mathrm{n}}$ & $4.63 \times 10^{-4}$ & $80{ }^{\circ} \mathrm{C}$ and $90 \% \mathrm{RH}$ & This work \\
\hline$\left.\left\{\left[\mathrm{Zn}\left(\mathrm{C}_{10} \mathrm{H}_{2} \mathrm{O}_{8}\right)_{0.5}\left(\mathrm{C}_{10} \mathrm{~S}_{2} \mathrm{~N}_{2} \mathrm{H}_{8}\right)\right] \cdot 2 \mathrm{H}_{2} \mathrm{O}\right]\right\}_{\mathrm{n}}$ & $4.39 \times 10^{-4}$ & $80{ }^{\circ} \mathrm{C}$ and $95 \% \mathrm{RH}$ & 4 \\
\hline$\left[\mathrm{Zn}(\mathrm{PDA})\left(\mathrm{H}_{2} \mathrm{MDP}\right)(\mathrm{MeOH})\left(\mathrm{H}_{2} \mathrm{O}\right)_{5}\right]_{\mathrm{n}}$ & $3.22 \times 10^{-4}$ & $40{ }^{\circ} \mathrm{C}$ and $90 \% \mathrm{RH}$ & This work \\
\hline $\mathrm{Zn}-\left(\mathrm{m}-\mathrm{H}_{6} \mathrm{~L}\right)$ & $1.39 \times 10^{-4}$ & $41^{\circ} \mathrm{C}$ and $98 \% \mathrm{RH}$ & 5 \\
\hline$\left.\left\{\mathrm{Cd} 2(\mathrm{DpmpcH}) \cdot \mathrm{H}_{2} \mathrm{O}\right) 2 \mathrm{Cl}_{2}\right\}_{\mathrm{n}}$ & $1.38 \times 10^{-4}$ & $50{ }^{\circ} \mathrm{C}$ and $97 \% \mathrm{RH}$ & 6 \\
\hline $\mathrm{Mg}-\left(\mathrm{p}-\mathrm{H}_{6} \mathrm{~L}\right)$ & $9.75 \times 10^{-5}$ & $41^{\circ} \mathrm{C}$ and $98 \% \mathrm{RH}$ & 5 \\
\hline$\left\{\left[\mathrm{Sr}\left(\mathrm{o}-\mathrm{CPhH}_{2} \mathrm{IDC}\right)\left(\mathrm{H}_{2} \mathrm{O}\right)_{2}\right] \cdot 2 \mathrm{H}_{2} \mathrm{O}\right\}_{n}$ & $6.08 \times 10^{-5}$ & $100{ }^{\circ} \mathrm{C}$ and $98 \% \mathrm{RH}$ & 7 \\
\hline$\left[\mathrm{Mn}\left(o-\mathrm{CPhH}_{2} \mathrm{IDC}\right)\left(4.4^{\prime} \mathrm{bipy}\right)_{0.5}\left(\mathrm{H}_{2} \mathrm{O}\right)_{2}\right]_{\mathrm{n}} \cdot 3 \mathrm{H}_{2} \mathrm{O}$ & $5.74 \times 10^{-5}$ & $100{ }^{\circ} \mathrm{C}$ and $98 \% \mathrm{RH}$ & 8 \\
\hline $\begin{array}{l}{\left[\mathrm{Zn}_{5}\left(o-\mathrm{CPhH}_{2} \mathrm{IDC}\right)_{2}(\mathrm{o}-\mathrm{CPhHIDC})_{2}\left(2.2^{\prime}-\right.\right.} \\
\left.\text { bipy })_{5}\right]_{\mathrm{n}} \cdot 5 \mathrm{H}_{2} \mathrm{O}\end{array}$ & $5.00 \times 10^{-5}$ & $100{ }^{\circ} \mathrm{C}$ and $100 \% \mathrm{RH}$ & 8 \\
\hline$\left(\mathrm{NH}_{4}\right)_{2}\left[\mathrm{Ag}_{4}(\mathrm{mel})\left(\mathrm{NH}_{3}\right)_{2}\right] \cdot 3 \mathrm{H}_{2} \mathrm{O}$ & $4.3 \times 10^{-5}$ & $85^{\circ} \mathrm{C}$ and $98 \% \mathrm{RH}$ & 9 \\
\hline$\left[\mathrm{Cu}_{4}(\mathrm{HDMPhIDC})_{4}\left(\mathrm{H}_{2} \mathrm{O}\right)_{4}\right]_{\mathrm{n}}$ & $2.58 \times 10^{-5}$ & $100{ }^{\circ} \mathrm{C}$ and $98 \% \mathrm{RH}$ & 10 \\
\hline$\left\{\left[\mathrm{Co}(2,6-\text { ndc })(\text { aldrithiol })\left(\mathrm{H}_{2} \mathrm{O}\right)_{2}\right] \cdot 2\left(\mathrm{H}_{2} \mathrm{O}\right)\right\}_{\mathrm{n}}$ & $1.96 \times 10^{-5}$ & $45{ }^{\circ} \mathrm{C}$ and $95 \% \mathrm{RH}$ & 11 \\
\hline$\left[\mathrm{Cu}_{2}(\mathrm{PDA})_{2}(\mathrm{Ald})_{2}(\mathrm{H} 2 \mathrm{O})_{2}\right] \cdot 8 \mathrm{H}_{2} \mathrm{O}$ & $1.19 \times 10^{-5}$ & $45^{\circ} \mathrm{C}$ and $100 \% \mathrm{RH}$ & 12 \\
\hline$\left.\left\{\left[\mathrm{Zn}\left(\mathrm{C}_{10} \mathrm{H}_{2} \mathrm{O}_{8}\right)_{0.5}\left(\mathrm{C}_{10} \mathrm{~S}_{2} \mathrm{~N}_{2} \mathrm{H}_{8}\right)\right] \cdot 5 \mathrm{H}_{2} \mathrm{O}\right]\right\}_{\mathrm{n}}$ & $2.55 \times 10^{-7}$ & $80{ }^{\circ} \mathrm{C}$ and $95 \% \mathrm{RH}$ & 4 \\
\hline$\left\{[\mathrm{Zn}(2,6-\mathrm{ndc})(\text { aldrithiol })] \cdot 3\left(\mathrm{H}_{2} \mathrm{O}\right)\right\}_{\mathrm{n}}$ & $6.73 \times 10^{-7}$ & $45^{\circ} \mathrm{C}$ and $95 \% \mathrm{RH}$ & 11 \\
\hline
\end{tabular}




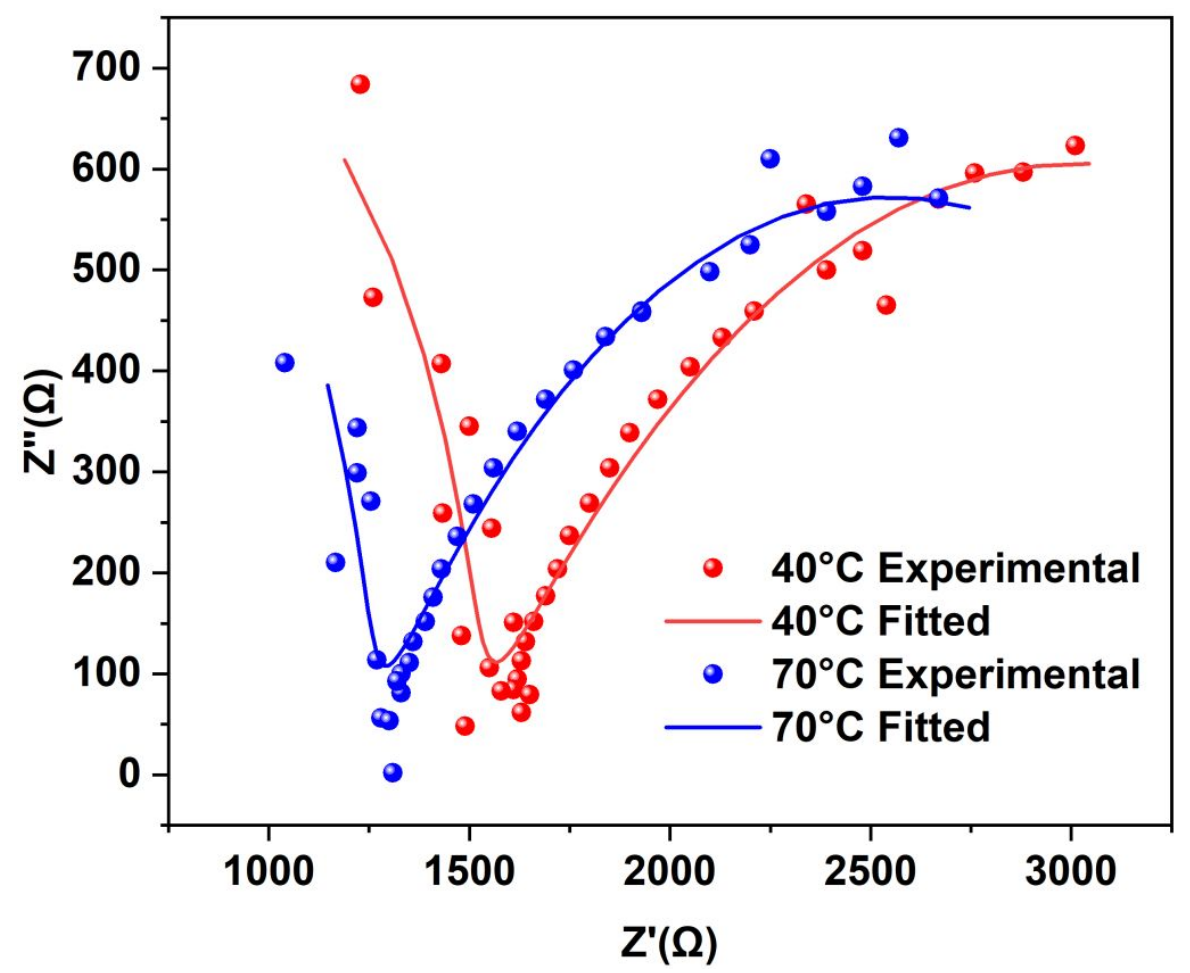

Figure. S14. Nyquist plots of impedance spectra of PDA-FA at $40^{\circ} \mathrm{C}$ and $70^{\circ} \mathrm{C}$ (at $33 \% \mathrm{RH}$ )

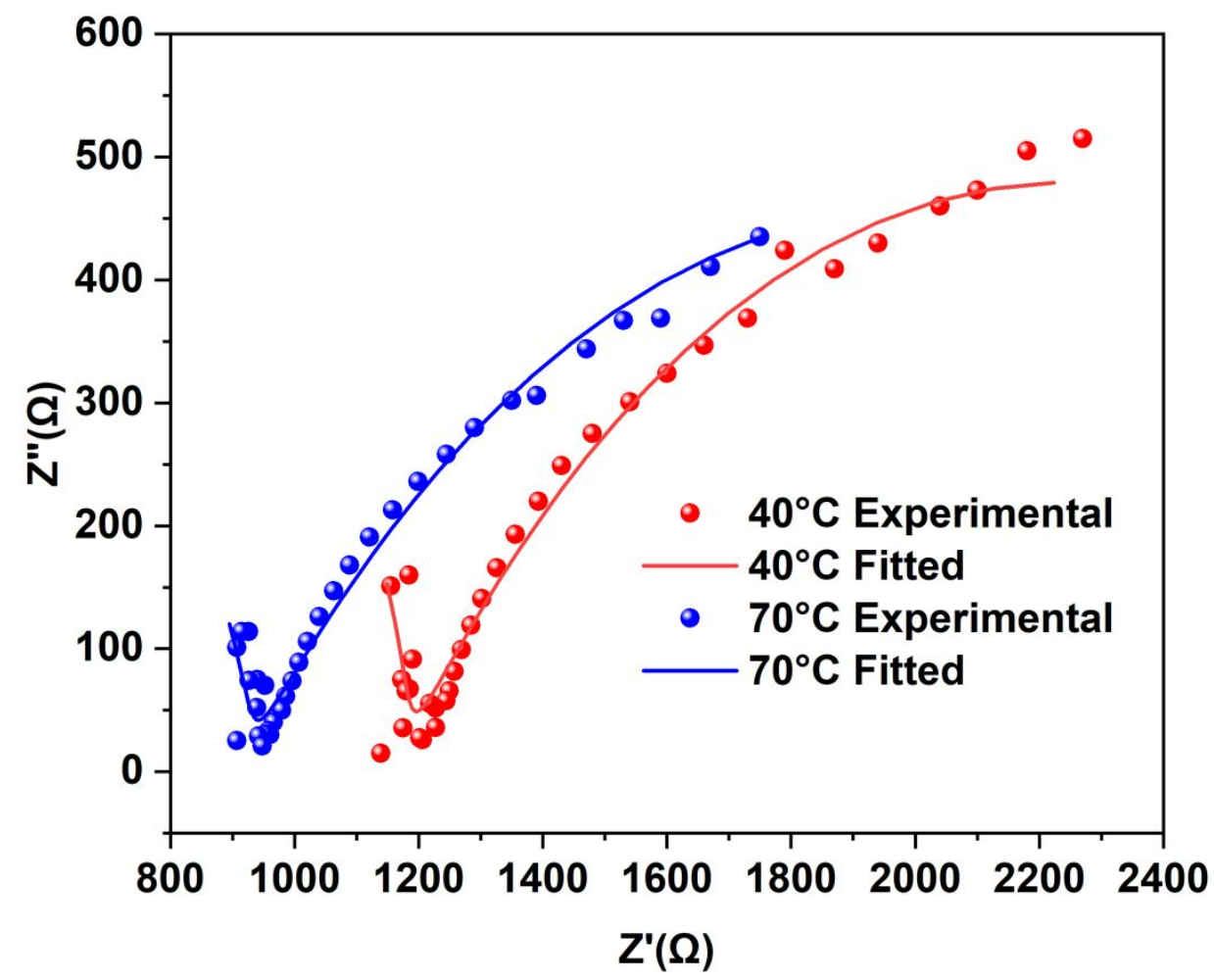

Figure. S15. Nyquist plots of impedance spectra of PDA-FA at $40^{\circ} \mathrm{C}$ and $70^{\circ} \mathrm{C}$ (at $75 \% \mathrm{RH}$ ) 


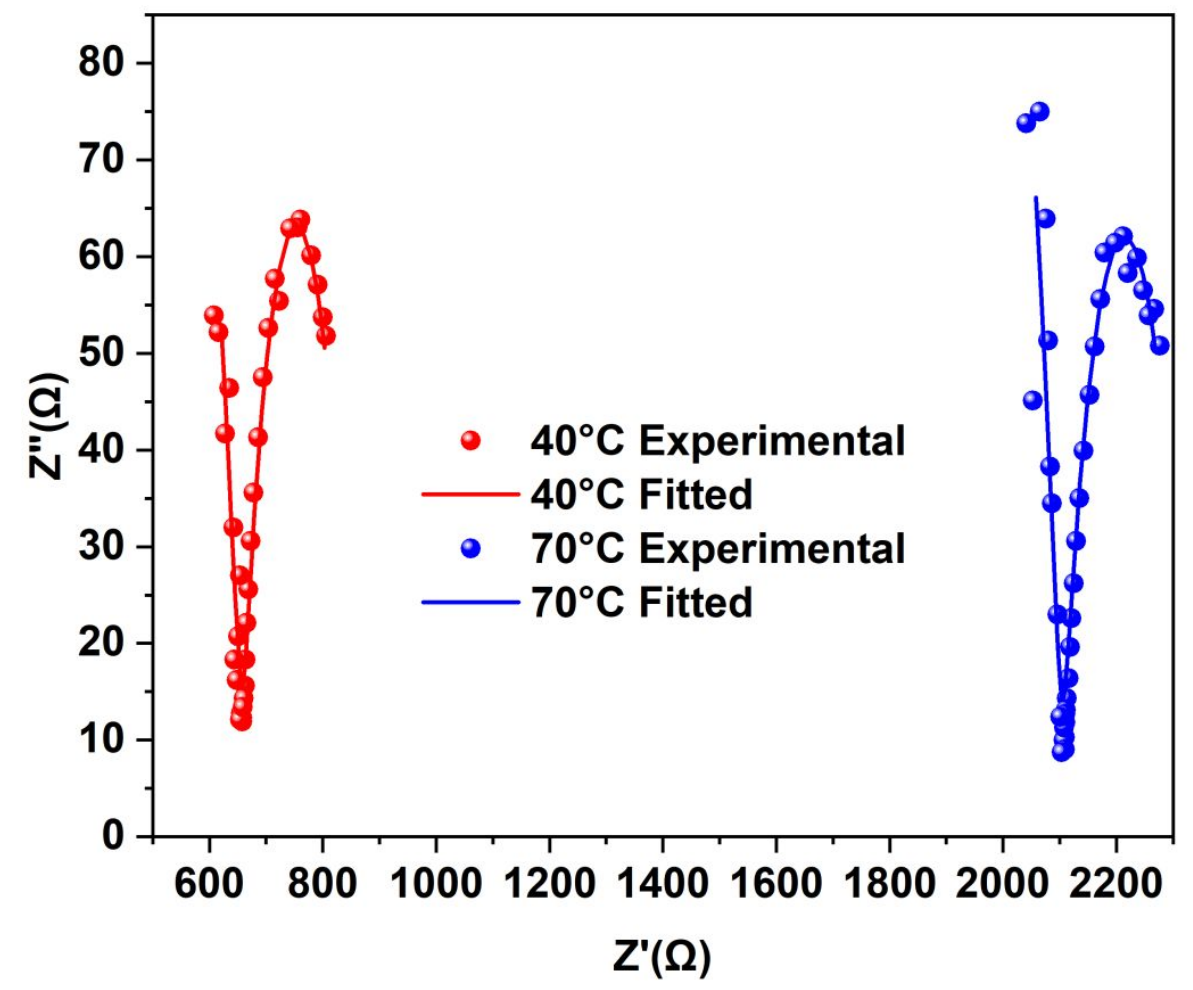

Figure. S16. Nyquist plots of impedance spectra of PDA-WC at $40^{\circ} \mathrm{C}$ and $70^{\circ} \mathrm{C}$ (at $33 \% \mathrm{RH}$ )

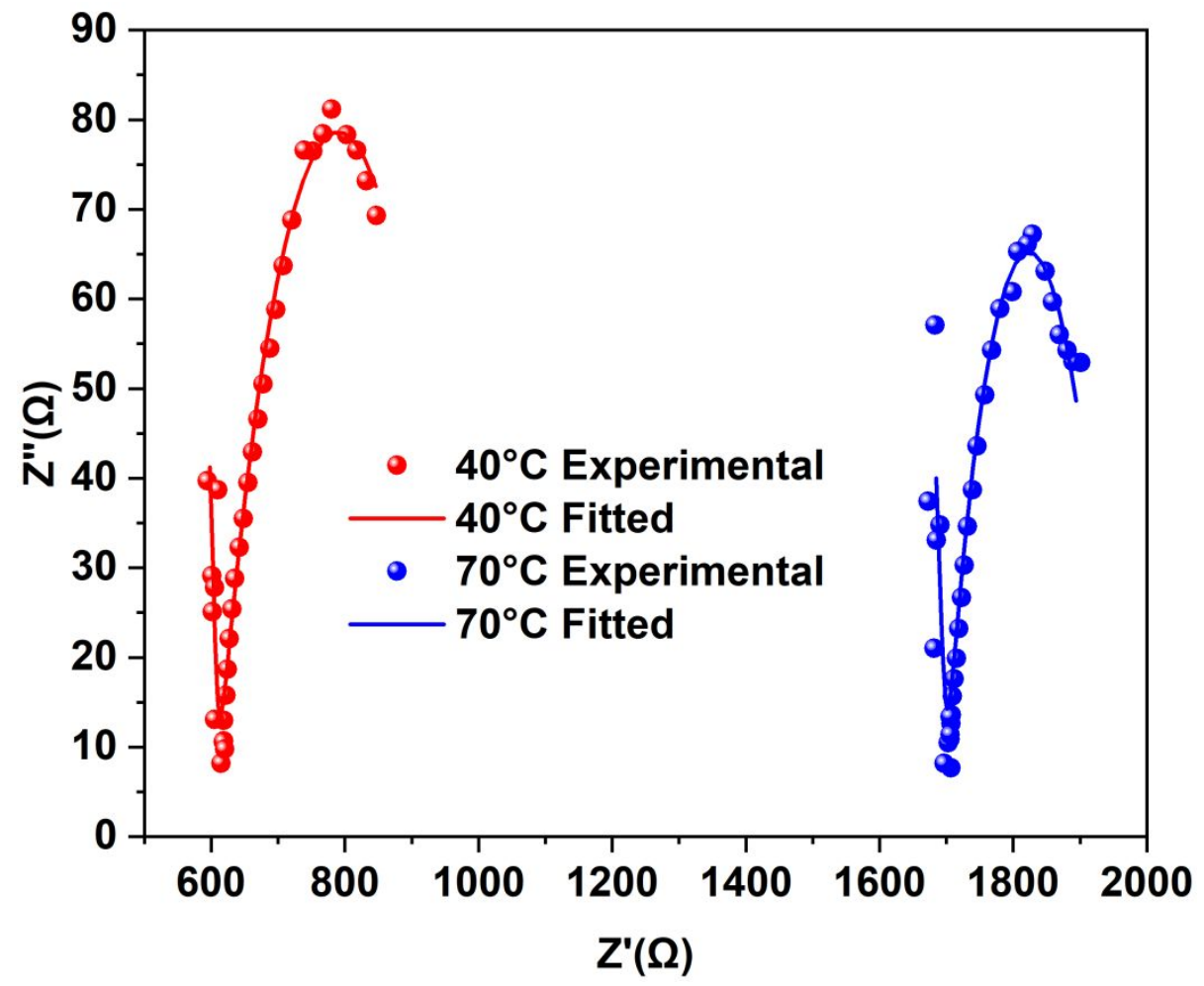

Figure. S17. Nyquist plots of impedance spectra of PDA-WC at $40^{\circ} \mathrm{C}$ and $70^{\circ} \mathrm{C}$ (at $75 \% \mathrm{RH}$ ) 


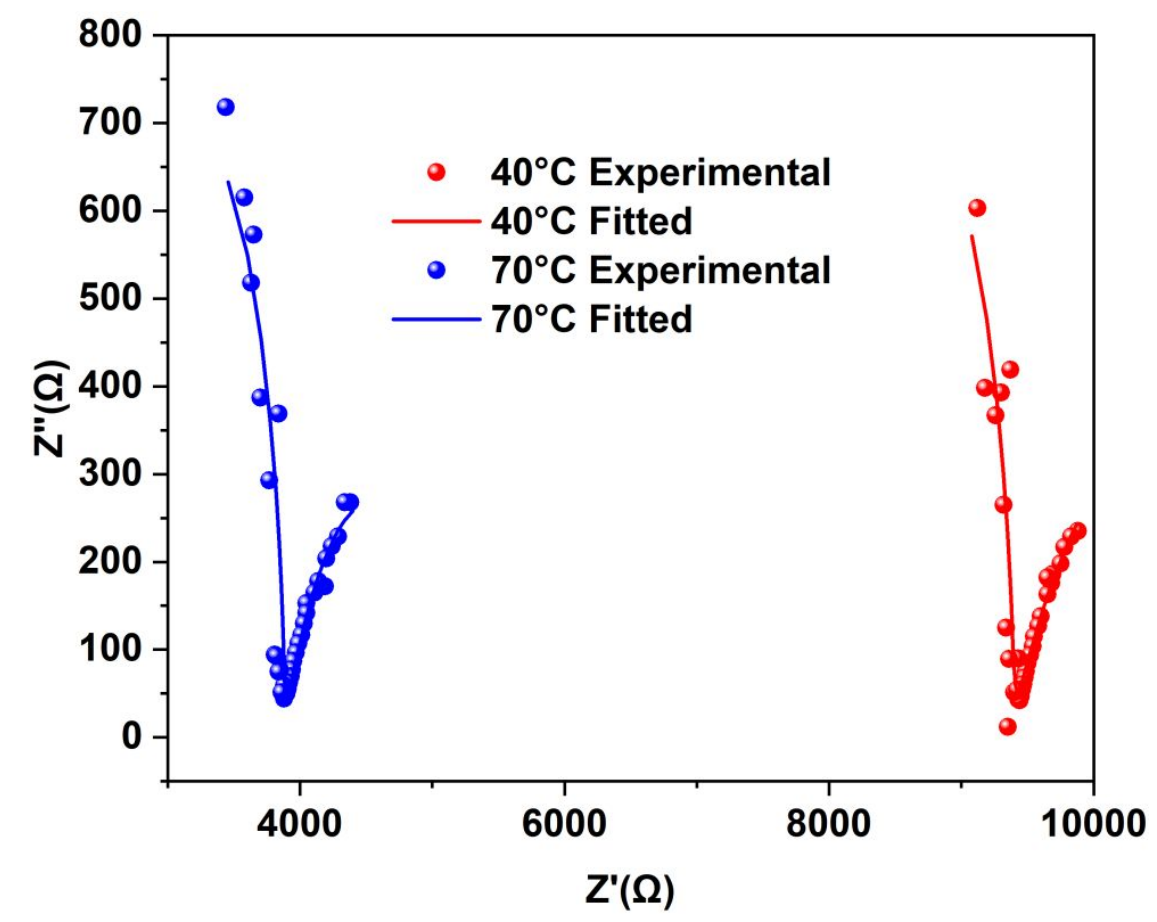

Figure. S18. Nyquist plots of impedance spectra of activated PDA-WC at $40^{\circ} \mathrm{C}$ and $70^{\circ} \mathrm{C}$ (at $33 \% \mathrm{RH})$

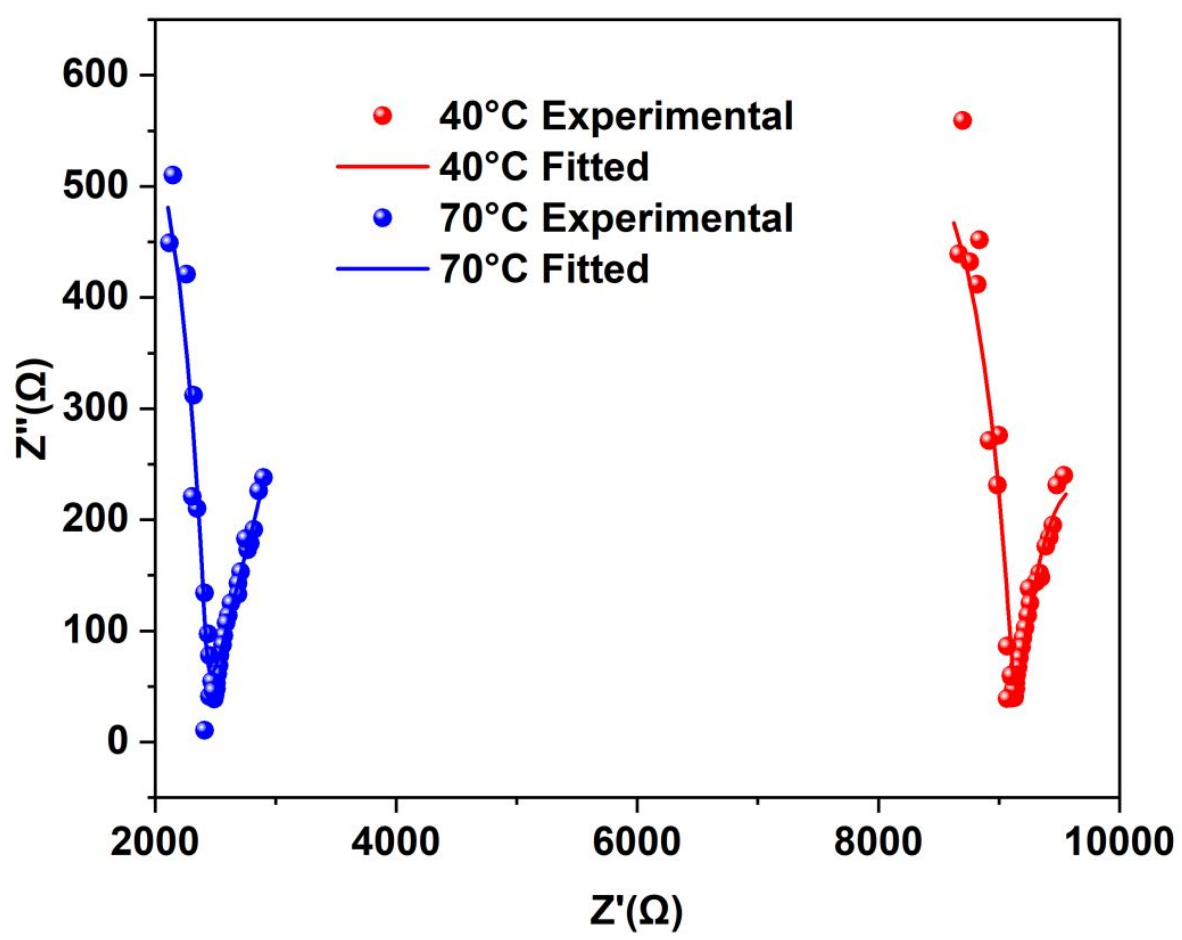

Figure. S19. Nyquist plots of impedance spectra of activated PDA-WC at $40^{\circ} \mathrm{C}$ and $70^{\circ} \mathrm{C}$ (at $75 \% \mathrm{RH})$ 


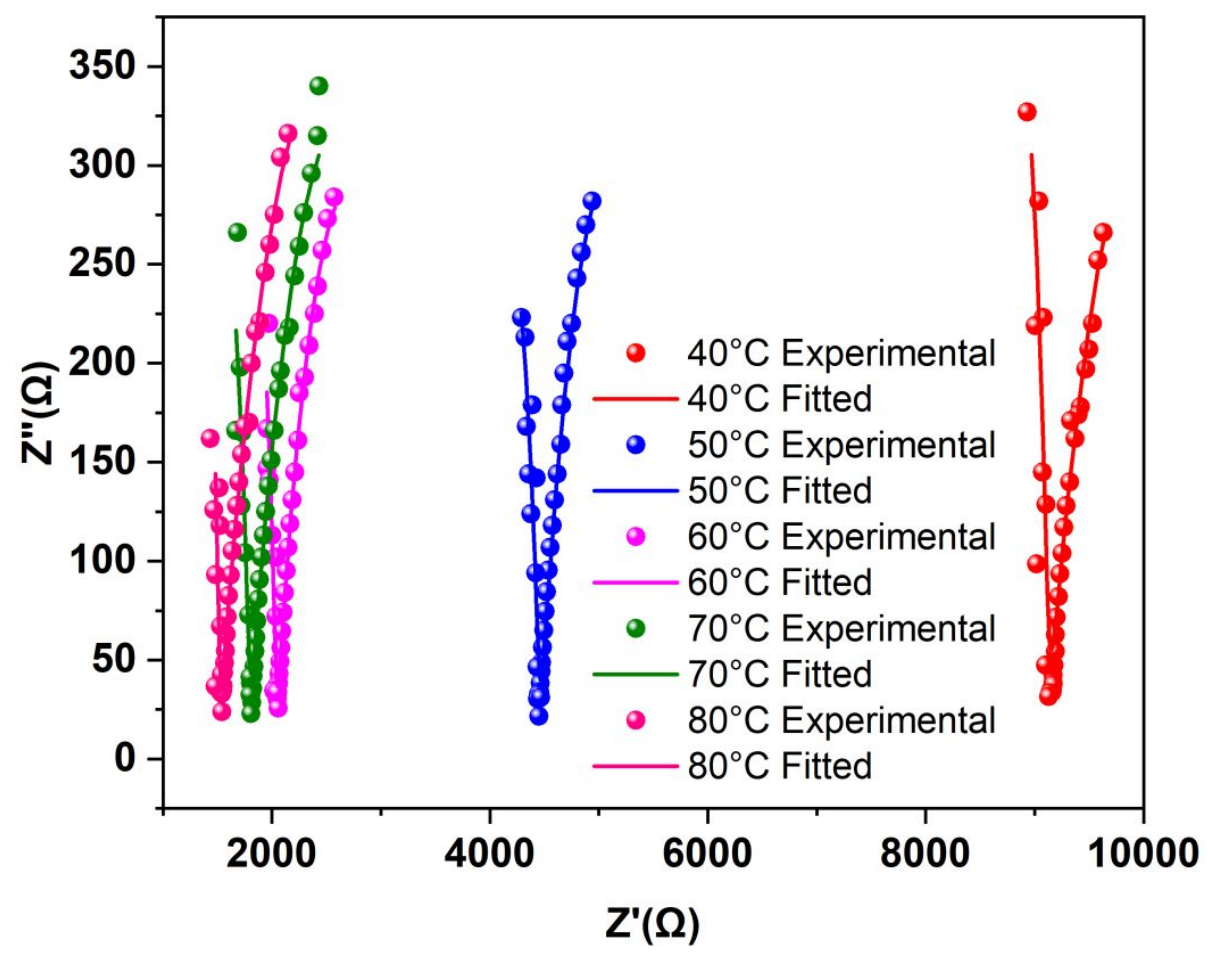

Figure. S20. Nyquist plots of impedance spectra of activated PDA-WC at $40^{\circ} \mathrm{C}, 50^{\circ} \mathrm{C}, 60^{\circ} \mathrm{C}$, $70^{\circ} \mathrm{C}$ and $80^{\circ} \mathrm{C}($ at $90 \% \mathrm{RH})$

\section{References}

1.Wang, M.; Luo, H.-B.; Liu, S.-X.; Zou, Y.; Tian, Z.-F.; Li, L.; Liu, J.-L.; Ren, X.-M., Water assisted high proton conductance in a highly thermally stable and superior water-stable openframework cobalt phosphate. Dalton Trans. 2016, 45, 19466-19472.

2.Qin, Y.; Xue, M.-H.; Dou, B.-H.; Sun, Z.-B.; Li, G., High protonic conduction in two metalorganic frameworks containing high-density carboxylic groups. New J. Chem. 2020, 44, 27412748.

3.Xu, H.; Feng, L.; Huang, W.; Wang, Q.; Zhou, H., A water-stable Ni(ii) diphosphonate exhibiting water vapor adsorption and water-assisted high proton conductivity. New J. Chem. 2019, 43, 807-812. 
4.Sahoo, S. C.; Kundu, T.; Banerjee, R., Helical Water Chain Mediated Proton Conductivity in Homochiral Metal-Organic Frameworks with Unprecedented Zeolitic unh-Topology. J. Am. Chem. Soc. 2011, 133, 17950-17958.

5.Colodrero, R. M. P.; Angeli, G. K.; Bazaga-Garcia, M.; Olivera-Pastor, P.; Villemin, D.; Losilla, E. R.; Martos, E. Q.; Hix, G. B.; Aranda, M. A. G.; Demadis, K. D.; Cabeza, A., Structural Variability in Multifunctional Metal Xylenediaminetetraphosphonate Hybrids. Inorg. Chem. 2013, 52, 8770-8783.

6.Liang, X.; Cai, K.; Zhang, F.; Liu, J.; Zhu, G., One, two, and three-dimensional metal-organic coordination polymers derived from enantiopure organic phosphorate: homochirality, water stability and proton conduction. CrystEngComm 2017,19, 6325-6332.

7.Shigematsu, A.; Yamada, T.; Kitagawa, H., Wide Control of Proton Conductivity in Porous Coordination Polymers. J. Am. Chem. Soc. 2011, 133, 2034-2036.

8.Zhu, M.; Hao, Z.-M.; Song, X.-Z.; Meng, X.; Zhao, S.-N.; Song, S.-Y.; Zhang, H.-J., A new type of double-chain based 3D lanthanide(iii) metal-organic framework demonstrating proton conduction and tunable emission. Chem. Commun. 2014, 50 (15), 1912-1914.

9.Dong, X.-Y.; Li, X.; Li, B.; Zhu, Y.-Y.; Zang, S.-Q.; Tang, M.-S., Water sandwiched by a pair of aromatic rings in a proton-conducting metal-organic framework. Dalton Trans. 2016, 45 (45), 18142-18146.

10.Liu, R.-L.; Shi, Z.-Q.; Wang, X.-Y.; Li, Z.-F.; Li, G., Two Highly Stable Proton Conductive Cobalt(II)-Organic Frameworks as Impedance Sensors for Formic Acid. Chem. Eur. J. 2019, 25 (62), 14108-14116.

11.Sanda, S.; Biswas, S.; Parshamoni, S.; Konar, S., Unraveling the multi-functional behavior in a series of Metal Organic Frameworks. J. Solid State Chem. 2015, 229, 103-111.

12.Sarkar, D.; Chandra Rao, P.; Aiyappa, H. B.; Kurungot, S.; Mandal, S.; Ramanujam, K.; Mandal, S., Multifunctional copper dimer: structure, band gap energy, catalysis, magnetism, oxygen reduction reaction and proton conductivity. RSC Adv. 2016, 6, 37515-37521. 